\title{
The $E_{\mathrm{p}}-E_{\mathrm{iso}}$ relation: intrinsic GRB property or/and selection effects?
}

\section{Robert Mochkovitch*}

Institut d'Astrophysique de Paris

E-mail: mochko@iap.fr

\section{Romain Hascoët}

Institut d'Astrophysique de Paris

E-mail: hascoeteiap.fr

\section{Frédéric Daigne}

Institut d'Astrophysique de Paris

E-mail: daignediap.fr

\section{Jean-Luc Atteia}

Institut de Recherche en Astrophysique et Planélogie - Observatoire Midi-Pyrénées

E-mail: Jean-luc.Atteia@irap.omp.eu

\section{Vincent Heussaff}

Institut de Recherche en Astrophysique et Planélogie - Observatoire Midi-Pyrénées

E-mail: vincent.heussaffefree.fr

\section{Sonila Bocí}

University of Tirana

E-mail: sonilaboci@yahoo.com

\section{Mimoza Hafizi}

University of Tirana

E-mail: mimoza.hafizi@fshn.edu.al

Since its discovery the $E_{\mathrm{p}}-E_{\mathrm{iso}}$ relation has been a source of controversy between those believing that it represents an intrinsic property of bursts that should be directly related to the physics of the prompt emission, and others arguing that it results from various selection effects (thresholds for burst and afterglow detection, for measuring the redshift, etc). In the context of the internal shock model we present a series of Monte-Carlo simulations showing that the observed relation may indeed come from a combination of intrinsic processes and selection effects. We find that the low $E_{\mathrm{p}}$ edge of the relation arises from the physics of shocks while the large $E_{\mathrm{p}}$ edge is mainly shaped by selection effects.

Gamma-Ray Bursts 2012 Conference-GRB2012,

May 07-11, 2012

Munich, Germany

\footnotetext{
*Speaker.
} 


\section{Introduction}

Following the first redshift determinations obtained thanks to the Beppo-SAX satellite [1], it became for the first time possible to address the issue of burst energetics from the estimate of $E_{\text {iso }}$, the total energy released in gamma-rays, assuming isotropic emission. Knowing the redshift also allowed to measure $E_{\mathrm{p}, 0}$, the peak energy of the spectrum in the burst rest frame. Using a limited sample of a dozen of events [2] found a correlation between these two quantities, with $E_{\mathrm{p}, 0} \propto E_{\text {iso }}^{1 / 2}$. This initial sample has now grown (using data from HETE 2 and Swift) to more than 100 bursts. The $E_{\mathrm{p}}-E_{\mathrm{iso}}$ correlation is confirmed in this extended sample and takes the form [3]

$$
E_{\mathrm{p}, 0}=110\left(\frac{E_{\text {iso }}}{10^{52} \mathrm{erg}}\right)^{0.55} \mathrm{keV} \quad \text { with a dispersion } \sigma=0.23 \mathrm{dex} .
$$

Since its discovery this relation has generated a long standing controversy with critics suggesting that it was largely shaped by selection effects. A key argument came from the observational $E_{\mathrm{p}, \mathrm{obs}}$ $F$ diagram (where $F$ is the burst fluence). It was shown by [4] that if Eq.(1.1) was exactly satisfied, all bursts should lie in the upper part of the diagram (i.e. should have $F>F_{\text {crit }}\left(E_{\mathrm{p}, \mathrm{obs}}\right)$ ). A test with BATSE data [5] shows that the fraction of outliers is large (up to 80\%) which seems to invalidate the $E_{\mathrm{p}}-E_{\text {iso }}$ relation. Including the possibility of a $3 \sigma$ dispersion however strongly improves the situation, reducing the fraction of outliers to less than $10 \%$ [6].

The issue is of great importance as it concerns both the physics of GRBs and their possible use as a tool for cosmology. If the $E_{\mathrm{p}}-E_{\text {iso }}$ relation has an intrinsic origin, it should be accounted for by models of the prompt emission. It might also be used to "standardize" GRBs with the aim to constrain the cosmological parameters, in addition to what is already done with type Ia supernovae.

The mechanism producing the prompt emission in GRBs is still debated. Three main possibilities have been discussed in the literature: (i) internal shocks; (ii) reconnection in magnetized outflows and (iii) Comptonized thermal emission. The origin of the $E_{\mathrm{p}}-E_{\text {iso }}$ relation has been briefly addressed in the framework of models (ii) and (iii) [7] while the present study focuses on internal shocks. We construct large samples of synthetic bursts using a toy model where the whole sequence of internal shoks is reduced to the collision of only two shells. We describe the model in Sect.2 and present our results in Sect.3. They are discussed in Sect.4, which is also the conclusion.

\section{The $E_{\mathrm{p}}-E_{\text {iso }}$ relation in the context of the internal shocks}

We address the problem of the $E_{\mathrm{p}}-E_{\text {iso }}$ relation in the context of internal shocks using an simplified two-shell toy model [8] that represents the whole sequence of internal shocks by the collision of only two spherical shells. Obviously this limitation prevents any realistic description of the resulting light curve but the global energetics of the model is preserved, which is the most important to discuss the $E_{\mathrm{p}}-E_{\text {iso }}$ relation. In particular, we expect that the $E_{\mathrm{p}}$ value from the toy model fairly represents the global $E_{\mathrm{p}}$ of a real burst averaged over the duration of the event.

The model parameters that fix the dynamics of the flow are the respective mass and Lorentz factor of the shells $\left(m_{i}, \Gamma_{i}\right)_{i=1,2}$ and the total duration $\tau$ of activity of the central engine. We can then define the average injected kinetic power, average Lorentz factor and contrast in the flow

$$
\dot{E}_{\mathrm{K}}=\frac{\left(m_{1} \Gamma_{1}+m_{2} \Gamma_{2}\right) c^{2}}{\tau} \quad \bar{\Gamma}=\frac{m_{1} \Gamma_{1}+m_{2} \Gamma_{2}}{m_{1}+m_{2}} \quad \kappa=\frac{\Gamma_{1}}{\Gamma_{2}} \quad\left(\Gamma_{1}>\Gamma_{2}\right) .
$$


The two shells collide and merge at a distance from the source

$$
R_{m}=2 c \tau \frac{\Gamma_{1}^{2} \Gamma_{2}^{2}}{\Gamma_{1}^{2}-\Gamma_{2}^{2}} \simeq 2 c \tau \Gamma_{2}^{2}
$$

and the energy dissipated in the collision is given by

$$
E_{\mathrm{diss}}=\left[m_{1} \Gamma_{1}+m_{2} \Gamma_{2}-\left(m_{1}+m_{2}\right) \Gamma_{m}\right] c^{2}=\dot{E}_{\mathrm{K}} \tau f_{\mu}(\kappa)
$$

where $\Gamma_{m}$ is the Lorentz factor of the merged shell after the collision and the function $f_{\mu}(\kappa)$ depends on the contrast only, for a given mass ratio $\mu=m_{1} / m_{2}$ (for example $f_{\mu}(\kappa)=\frac{\kappa+1-2 \sqrt{\kappa}}{\kappa+1}$ for $\mu=1$ ).

We assume that a fraction $\varepsilon_{e}$ of the dissipated energy is injected into shock-accelerated electrons and is efficiently radiated by the synchrotron process (so that $E_{\text {iso }}=\varepsilon_{e} E_{\mathrm{diss}}$ ). The resulting spectrum is a broken power law with a break at the synchrotron energy [8]

$$
E_{\mathrm{syn}}=K_{s} B \gamma_{e}^{2} \Gamma_{m}=F\left(\varepsilon_{e}, \varepsilon_{B}, \zeta\right) \times \frac{\dot{E}_{\mathrm{K}}^{1 / 2} \varphi(\kappa)}{\tau \bar{\Gamma}^{2}}
$$

where $K_{s}=\frac{3}{2} \frac{e \hbar}{m_{e} c}$ is the synchrotron constant, $\gamma_{e}$ the characteristic Lorentz factor of the accelerated electrons and $B$ the shock-amplified magnetic field. The values of $\gamma_{e}$ and $B$ depend both on the flow dynamics (via the comoving dissipated energy per unit mass and unit volume) and the microphysics parameters $\varepsilon_{e}, \varepsilon_{B}$ and $\zeta$ (the fraction of electrons which are accelerated).

The simplicity of the two-shell approach allows to construct large synthetic burst samples to test the $E_{\mathrm{p}}-E_{\text {iso }}$ relation. We perform a Monte-Carlo simulation with the following distributions of the model parameters:

- The injected power $\dot{E}_{\mathrm{K}}$ follows a power-law of index $\lambda=-1.7$ from $\dot{E}_{\mathrm{K}, \min } \lesssim 10^{52} \mathrm{erg} . \mathrm{s}^{-1}$ to $\dot{E}_{\mathrm{K}, \max } \gtrsim 10^{54}$ erg.s ${ }^{-1}$.

- The duration $\tau$ has a log-normal distribution (we only consider long GRBs). Since the typical variability timescale $\tau_{\mathrm{v}}$ in GRB light curves is generally smaller than the burst duration (except for single pulse events) we have computed the shock radius with $\tau_{\mathrm{v}}$ replacing $\tau$ in Eq.(2.2). We have tried various parametrization of the relation $\tau_{\mathrm{v}}(\tau)$. The results in Sect.(3.1) below are obtained with

$$
\begin{array}{ll}
\tau_{\mathrm{v}}(\tau)=\tau & \text { if } \tau \leq 1 \mathrm{~s} \\
\tau_{\mathrm{v}}(\tau)=\tau^{1 / 2} & \text { if } \tau>1 \mathrm{~s}
\end{array}
$$

- The average Lorentz factor $\bar{\Gamma}$ and the contrast $\kappa$ are uniformly distributed respectively from 100 to 300 and from 2 to 6 .

- The function $F\left(\varepsilon_{e}, \varepsilon_{B}, \zeta\right)$ is allowed to vary by no more than one order of magnitude. Since we have [8]

$$
F\left(\varepsilon_{e}, \varepsilon_{B}, \zeta\right) \propto \varepsilon_{B}^{1 / 2}\left(\frac{\varepsilon_{e}}{\zeta}\right)^{2}
$$

this implicitely assumes that the microphysics parameters (especially $\varepsilon_{e}$ and $\zeta$ ) do not vary strongly from burst to burst.

- The burst rate $R_{\mathrm{GRB}}(z)$ is not directly proportional to the SFR at large $z$ [9]. We have $R_{\mathrm{GRB}}(z)=$ $k(z) \times \operatorname{SFR}(z)$ with $k(z)$ being an increasing function of $z$. 

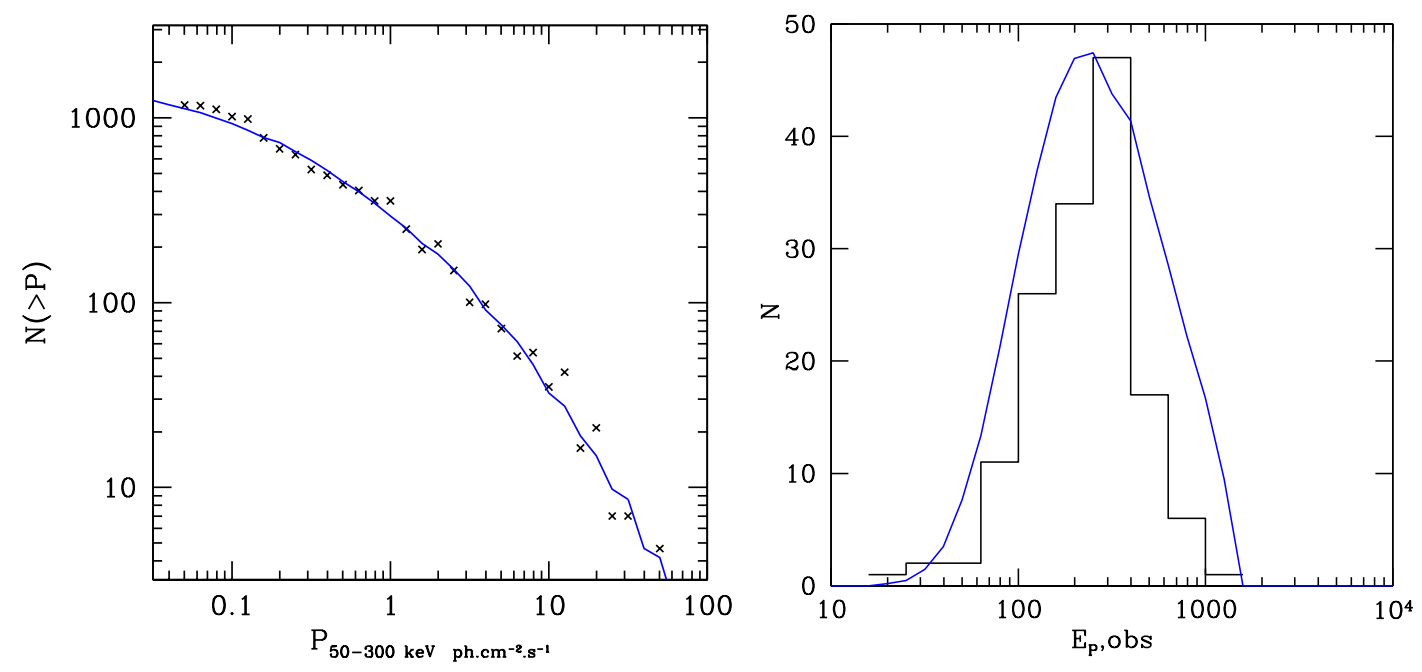

Figure 1: Left panel: $\log N-\log P$ relation obtained from the Monte-Carlo simulation (blue line) compared to the BATSE data collected by [10]; Right panel: Distribution of the observed $E_{\mathrm{p}}$ for bursts with a peak photon flux larger than $5 \mathrm{ph} . \mathrm{cm}^{-2} \cdot \mathrm{s}^{-1}$ in the $50-300 \mathrm{keV}$ energy range. The results of the simulation (in blue) are compared to the data from [11].

We impose that the simulation satisfies a set of four observational constraints: (i) the $\log N$ $\log P$ diagram of BATSE bursts [10]; (ii) the observed $E_{\mathrm{p}}$ distribution of bright BATSE bursts [11]; (iii) the duration distribution of long BATSE bursts [12]; (iv) the redshift distribution of Swift bursts [13]. They partially limit the range allowed for the model parameters. For example (i) constraints the index of the power-law distribution of $\dot{E}_{\mathrm{K}}$ to $\lambda=-1.7 \pm 0.2$. We illustrate in Fig.1 how the simulation fulfills constraints $(i)$ and (ii).

\section{Results}

The possibility to link $E_{\mathrm{p}}$ and $E_{\text {iso }}$ in the internal shock framework clearly appears when Eq.(2.3) is substituted into Eq.(2.4) to give

$$
E_{\text {syn }}=F^{\prime}\left(\varepsilon_{e}, \varepsilon_{B}, \zeta\right) \frac{G(\kappa)}{\tau^{1 / 2} \tau_{\mathrm{v}}(\tau) \bar{\Gamma}^{2}} \times E_{\text {iso }}^{1 / 2}=\mathscr{F}(\text { microphysics, } \kappa, \bar{\Gamma}, \tau) \times E_{\text {iso }}^{1 / 2}
$$

with $F^{\prime}=F / \varepsilon_{e}^{1 / 2}$ and $G=\varphi / f_{\mu}^{1 / 2}$. Eq.(3.1) would exactly be the Amati relation if the microphysics and outflow parameters (apart from $\dot{E}_{\mathrm{K}}$ ) were the same in all bursts. This is naturally not the case, which leads to a noticeable amount of dispersion. The black dots in the left panel of Fig. 2 represent a sample of $10^{5}$ synthetic events obtained with the assumptions on the model parameters discussed in Sect.2. They do not populate the $E_{\mathrm{p}}-E_{\mathrm{iso}}$ diagram randomly but define a sequence that remains much broader than the observed $E_{\mathrm{p}}-E_{\mathrm{iso}}$ relation. An interesting result however is that the model does not predict bursts with a large $E_{\text {iso }}$ and a low $E_{\mathrm{p}}$, in agreement with observations. Such events, producing many photons, would be easy to detect so that their absence cannot be the result of selection effects and must have a physical origin. 

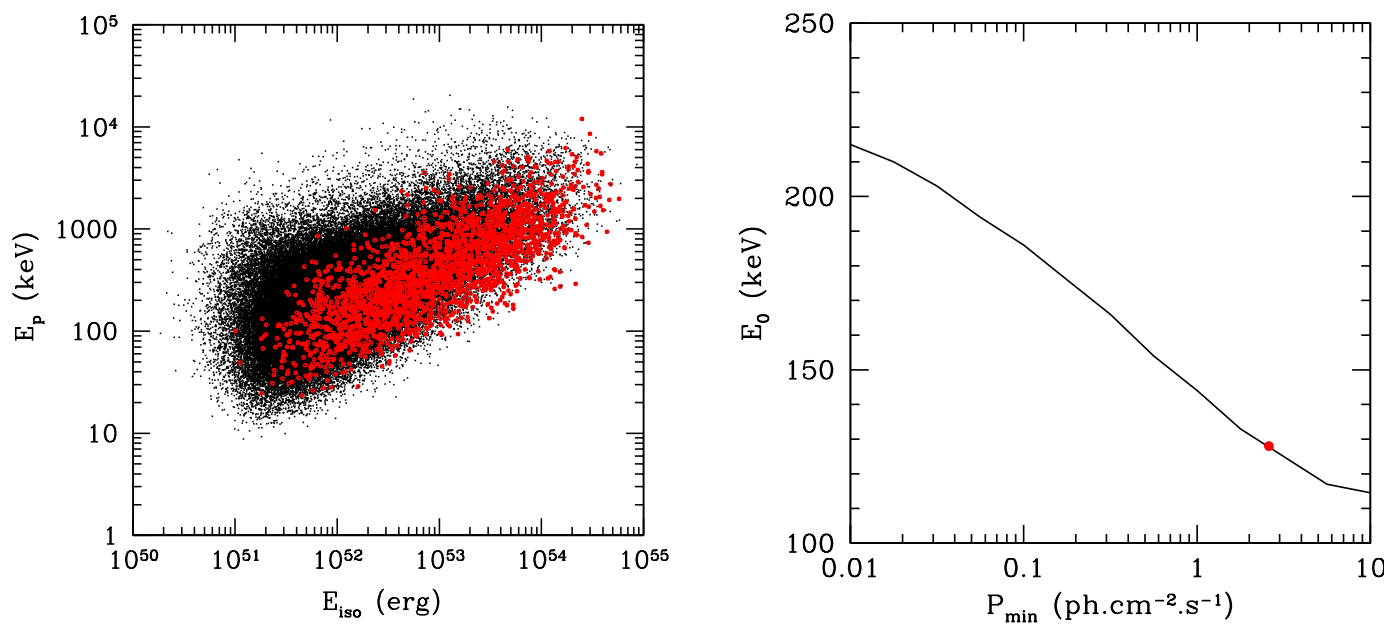

Figure 2: Left panel: $E_{\mathrm{p}}-E_{\text {iso }}$ diagram of $10^{5}$ synthetic bursts (black dots). The subsample of events with a peak flux larger than $2.6 \mathrm{ph} . \mathrm{cm}^{-2} \cdot \mathrm{s}^{-1}$ in the $15-150 \mathrm{keV}$ energy range is represented by red dots; Right panel: Normalization of the $E_{\mathrm{p}}-E_{\text {iso }}$ relation (defined as the value $E_{0}$ of $E_{\mathrm{p}}$ for $E_{\mathrm{iso}}=10^{52} \mathrm{erg}$ ) as a function of detector sensitivity in the $15-150 \mathrm{keV}$ energy range (the red dot corresponds to $2.6 \mathrm{ph} . \mathrm{cm}^{-2} . \mathrm{s}^{-1}$ ).

We introduce selection effects by imposing a threshold in photon flux at $2.6 \mathrm{ph} . \mathrm{cm}^{-2} \cdot \mathrm{s}^{-1}$ as in the nearly complete sample presented in [14]. Bursts above the threshold are shown as red dots in Fig.2. These detected events form a sequence very similar to the observed $E_{\mathrm{p}}-E_{\text {iso }}$ relation, with just a slightly larger dispersion $\sigma=0.3$ dex.

The existence of a large population of undetected events making a broader sequence than the presently observed $E_{\mathrm{p}}-E_{\text {iso }}$ relation should manifest itself with an instrument of increased sensitivity. When the threshold for burst detection is lowered, the dispersion and normalization $E_{0}$ of the relation ( $E_{0}$ being defined as the value of $E_{\mathrm{p}}$ for $E_{\text {iso }}=10^{52} \mathrm{erg}$ ) should increase. The right panel of Fig.2 illustrates this effect for $E_{0}$.

\section{Discussion and Conclusion}

To study the $E_{\mathrm{p}}-E_{\text {iso }}$ correlation in the context of internal shocks we used a Monte-Carlo approach to generate large samples of bursts with specific distributions of the model parameters (hydrodynamics and microphysics). We have obtained the following results:

- The GRB population defines a broad sequence in the $E_{\mathrm{p}}-E_{\text {iso }}$ diagram.

- The low $E_{\mathrm{p}}$ edge of the sequence is consistent with the observed $E_{\mathrm{p}}-E_{\mathrm{iso}}$ correlation (with no event with a large $E_{\text {iso }}$ and a low $E_{\mathrm{p}}$.

- When selection effects are included in the analysis (a simple threshold on the peak photon flux) they affect the large $E_{\mathrm{p}}$ edge of the sequence, leading to a $E_{\mathrm{p}}-E_{\text {iso }}$ relation very similar to the observed one. 
Our results however depend on the assumptions made for the distribution of the model parameters. Our choices are reasonable but we nevertheless plan to study the parameter space extensively, introducing for example possible coupling between variables (with e.g. the average Lorentz factor of the flow depending on the injected kinetic power). Other future developments will include a more careful discussion of the threshold condition (with limits on $E_{\mathrm{p}}$, on photon fluence, etc) and an extension of the study to the Yonetoku $\left(E_{\mathrm{p}}-L_{\text {iso }}\right)$ correlation.

\section{References}

[1] M.R. Metzger, S.G. Djorgovski, S.R. Kulkarni, et al., Spectral constraints on the redshift of the optical counterpart to the Gamma-ray burst of 8 May 1997, Nature 387 (1997) 878

[2] L. Amati, F. Frontera, M. Tavani, et al., Intrinsic spectra and energetics of BeppoSAX Gamma-Ray Bursts with known redshifts, A\&A 390 (2002)81 [astro-ph / 0205230].

[3] L. Amati, The Ep,i - Eiso correlation and Fermi Gamma-Ray Bursts, astro-ph/1002.2232.

[4] E. Nakar, T. Piran Outliers to the peak energy-isotropic energy relation in gamma-ray bursts, MNRAS 360 (2005)L73 [astro-ph/ 0501559 ].

[5] D.L. Band, R.D. Preece Testing the Gamma-Ray Burst Energy Relationships, ApJ 627 (2005) 319 [astro-ph/1002.2232].

[6] L. Nava, G. Ghirlanda, G. Ghisellini, et al., Fermi/GBM and BATSE gamma-ray bursts: comparison of the spectral properties, MNRAS 415 (2011) 3153 [astro-ph/1012 . 3968].

[7] D. Giannios The peak energy of dissipative gamma-ray burst photospheres, MNRAS 422 (2012) 3092 [astro-ph/1111.4258].

[8] C. Barraud, F. Daigne, R. Mochkovitch, J.L. Atteia, On the nature of X-ray flashes, A\&A 440 (2005) 809 [astro-ph/0507173].

[9] F. Daigne, E.M. Rossi, R. Mochkovitch, The redshift distribution of Swift gamma-ray bursts: evidence for evolution, MNRAS 372 (2006) 1034 [ast ro-ph / 0607618 ].

[10] B.E. Stern, Y. Tikhomirova, M. Stepanov, et al., A Search for Nontriggered Gamma-Ray Bursts in the BATSE Continuous Records: First Results, ApJ 540 (2000) L21 [astro-ph/9903094].

[11] R.D. Preece, M.S. Briggs, R.S. Mallozzi, et al., The BATSE Gamma-Ray Burst Spectral Catalog. I. High Time Resolution Spectroscopy of Bright Bursts Using High Energy Resolution Data, ApJS 126 (2000) 19 [astro-ph/9908119].

[12] C. Kouveliotou, C.A. Meegan, G.D. Fishman, et al., Identification of two classes of gamma-ray bursts, ApJ 413 (1993) L101.

[13] P. Jakobsson, A. Levan, J.P.U. Fynbo, et al., A mean redshift of 2.8 for Swift gamma-ray bursts, A\&A 447 (2006) 897 [astro-ph/ 0509888 ].

[14] R. Salvaterra, S. Campana, S. Vergani, et al., A Complete Sample of Bright Swift Long Gamma-Ray Bursts. I. Sample Presentation, Luminosity Function and Evolution, ApJ 749 (2012) 68 [astro-ph/1112.1700]. 